\title{
On the chemical composition of cosmic rays of highest energy
}

\author{
Grzegorz Wilk \\ The Andrzej Soltan Institute for Nuclear Studies, Hoża 69, 00681, Warsaw, Poland \\ E-mail: wilk@fuw.edu.pl \\ Zbigniew Włodarczyk \\ Institute of Physics, Jan Kochanowski University, Świȩtokrzyska 15, 25-406 Kielce, \\ Poland \\ E-mail: zbigniew.wlodarczyk@ujk.kielce.pl
}

\begin{abstract}
We present arguments aiming at reconciling apparently contradictory results concerning the chemical composition of cosmic rays of highest energy, coming recently from the Auger and HiRes collaborations. In particular, we argue that the energy dependence of the mean value and root mean square fluctuation of shower maxima distributions observed by the Auger experiment are not necessarily caused by the change of nuclear composition of primary cosmic rays. They could also be caused by the change of distribution of the first interaction point in the cascade. A new observable, in which this influence is strongly suppressed, is proposed and tested.

PACS numbers: 96.50.sd, 96.50.sb, 13.85.Tp
\end{abstract}


Recently two leading cosmic ray (CR) experiments, the Pierre Auger Collaboration (Auger) [1] and The High Resolution Fly's Eye Collaboration (HiRes) [2] published their most recent data on the depth of maxima of extensive air showers above $10^{18}$ eV. Two apparently contradictory conclusions were presented. Whereas the Auger collaboration cautiously concluded that their data indicated a gradual increase of the average mass of incoming CR with energy, HiRes stated that their data were consistent with a predominantly protonic composition of cosmic rays. These results started a vivid discussion [3]. In this note, we propose a possible reconciliation of both results with an indication that, perhaps, there is no need to introduce a heavy (i.e., iron) component in the CR chemical composition. Namely, we indicate that these results could also be due to the influence of the distribution of the first interaction point in the cascade, at least partially. We therefore propose and test a new observable, cf. Eq. (9), in which this influence is strongly suppressed.

With increasing energy the Auger data [1] show almost monotonic changes in the chemical composition of CR changing from proton to iron for two types of observables considered: the mean depth of the maximum of the longitudinal development of air showers, $\left\langle X_{\max }\right\rangle$, and the shower-to-shower fluctuations, the root mean square (rms) $\sigma\left(X_{\max }\right)$, see Fig. $1 \pm$. For $\left\langle X_{\max }\right\rangle$, a such dependence can be interpreted by allowing for the presence of two components in CR: iron, with relative abundance $\alpha$, and protons, with relative abundance $1-\alpha$ :

$$
\left\langle X_{\max }\right\rangle=(1-\alpha)\left\langle X_{\max }\right\rangle_{p}+\alpha\left\langle X_{\max }\right\rangle_{F e}
$$

(where $\left\langle X_{\max }\right\rangle_{p}$ and $\left\langle X_{\max }\right\rangle_{F e}$ denote the mean depth of shower maxima for the pure $p$ and $F e$ CR's, respectively). However, the same reasoning applied to $\sigma\left(\left\langle X_{\max }\right\rangle\right)$ lead to nonmonotonic dependence on $\alpha$ in this case (seen as nonlinear spacing between lines corresponding to different values of $\alpha$, cf. Fig. $1 \mathrm{~b}$ ),

$$
\sigma^{2}=(1-\alpha) \sigma_{p}^{2}+\alpha \sigma_{F e}^{2}+\alpha(1-\alpha)\left(\left\langle X_{\max }\right\rangle_{p}-\left\langle X_{\max }\right\rangle_{F e}\right)^{2} .
$$

This has a maximum at

$$
\alpha=\frac{1}{2}\left[1-\frac{\sigma_{p}^{2}-\sigma_{F e}^{2}}{\left(\left\langle X_{\max }\right\rangle_{p}-\left\langle X_{\max }\right\rangle_{F e}\right)^{2}}\right] .
$$

This is seen in Fig. 1b, where one observes that adding iron to protons results first (for small $\alpha$ ) in increased fluctuations, and only for a quite large admixture of iron (large $\alpha$ ) do they decrease towards the pure iron line. For this reason, as seen in Fig. 2,

$\ddagger$ In all figures presented here we use both experimental data and model predictions for pure proton and iron primaries following [1, 2]. Using information on their values of $\langle X\rangle_{p},\langle X\rangle_{F e}, \sigma_{p}$ and $\sigma_{F e}$, one can deduce from Eqs.(11) and (2), in an univocal way, the corresponding values of observables of interest for a given value of the parameter $\alpha$; in particular, the energy dependencies of $\left\langle X_{\max }\right\rangle$ and $\sigma\left(X_{\max }\right)$. The differences $\left\langle X_{\max }\right\rangle-\sigma\left(X_{\max }\right)$ are evaluated directly from the definition using the energy dependencies of $\left\langle X_{\max }\right\rangle$ and $\sigma\left(X_{\max }\right)$ as given by the models. Notice that Auger compares their data with pure simulations, whereas HiRes quotes data including all detector effects and compares them to the models after the detector simulation. It means then that, unfortunately, both approaches cannot be compared directly. 
for such a simple parametrization, experimental data with similar energy behavior lead to quite different chemical compositions, ranging from proton dominated for $\left\langle X_{\max }\right\rangle$ to iron dominated for $\sigma\left(X_{\max }\right)$.

Among possible reasons of such a discrepancy, we shall concentrate on problems connected with the development of the cascade, in particular on the significance of the depth of the first interaction. It is known (cf. [5, 6, 7]) that most charged particles in the shower are electrons and positrons with energies near the critical energy $\varepsilon$ originated from the electromagnetic subshowers initiated by photons from $\pi^{0}$ decay. The mean depth of the maximum of an electromagnetic shower initiated by a photon with energy $E_{\gamma}$ is

$$
\left\langle X_{\max }^{\text {em }}\left(E_{\gamma}\right)\right\rangle=X_{0} \ln \left(\frac{E_{\gamma}}{\varepsilon}\right) .
$$

Here $X_{0} \approx 37 \mathrm{~g} / \mathrm{cm}^{2}$ is the radiation length and $\varepsilon=81 \mathrm{MeV}$ in the air. A nuclearinitiated shower consists of a hadronic core which feeds the electromagnetic component mainly through the production of $\pi^{0}$. Therefore, in general, for an incident nucleus of mass $A$ (including protons with $A=1$ ) and total energy $E$, the depth of the shower maximum is given by

$$
\left\langle X_{\max }\right\rangle=\left\langle X_{\max }^{e m}[(E / A)(K /\langle n\rangle)]\right\rangle+\left\langle X_{1}\right\rangle,
$$

where $\left\langle X_{1}\right\rangle$ is the mean depth of the interaction with maximal energy deposition into the shower (known also as the depth of the first interaction), $K$ denotes inelasticity and $\langle n\rangle$ is related to the multiplicity of secondaries produced in the high-energy hadronic interactions in the cascade. When the composition changes with energy, $\langle A\rangle$ depends on energy and $\left\langle X_{\max }\right\rangle$ changes accordingly. For primary nuclei with mass number $A$ and energy $E$, the shower is, within a good approximation, simply equivalent to a bundle of $A$ nucleons with energies $E / A$ each. In the case of primary protons in the hadronic cascade, there is a hierarchy of energies of secondary particles in each interaction and a similar (approximately geometrical) hierarchy of interaction energies in the cascade. In this case $\langle n\rangle$ has to be understood as some kind of effective multiplicity without a general straightforward definition. In addition, the inelasticity $K$ can also change with energy [8].

Now, the probability of observing the first interaction in a shower at a depth greater than $X$ is

$$
P\left(X_{1}>X\right) \propto \exp (-X / \lambda),
$$

where $\lambda$ denotes the interaction length (and is therefore connected to the cross section, in our case $\lambda_{p-a i r}=24160 / \sigma_{p-a i r}\left[\mathrm{~g} / \mathrm{cm}^{2}\right]$ for cross-section given in [mb]). It is tempting to use directly the exponential distributions of showers with large $X_{\max }$ to calculate $X_{1}$ (and the proton-air cross section). However, this can be done only in the case of a perfect correlation between $X_{\max }$ and $X_{1}$. The fluctuations existing in shower development modify such a relation, leading to

$$
P\left(X_{\max }>X\right) \propto \exp (-X / \Lambda)
$$



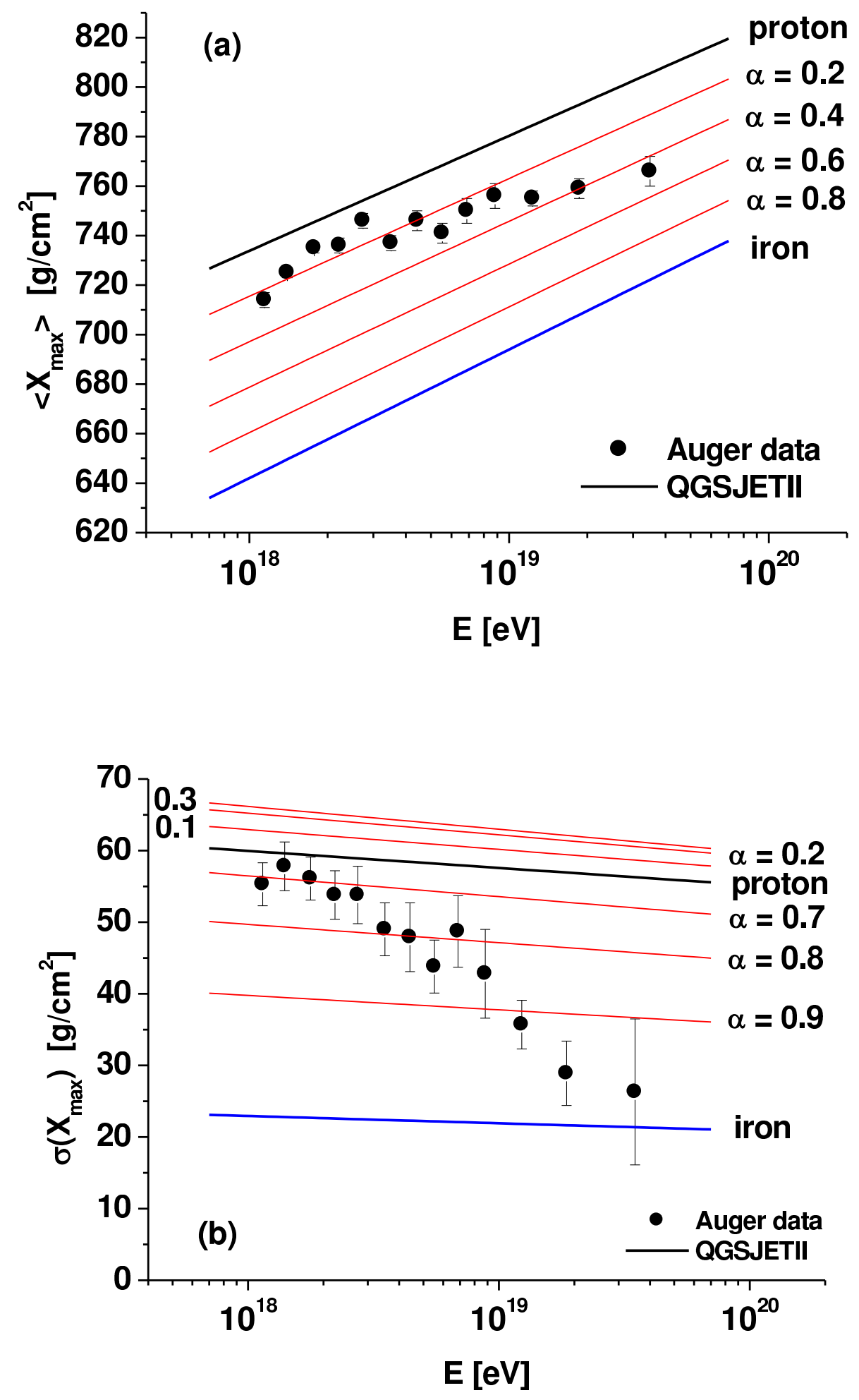

Figure 1. (Color online) $\left\langle X_{\max }\right\rangle$ and $\sigma\left(X_{\max }\right)$ from [1] compared with the QGSJETII model [4] using two components, protons and iron, cf. Eqs. (11) and (2), respectively, with $\alpha$ denoting the relative abundance of iron nuclei.

where $\Lambda=k \lambda$, and $k$ accounts for the way the energy dissipation takes place in the early stages of shower evolution; it is particularly sensitive to the mean inelasticity 


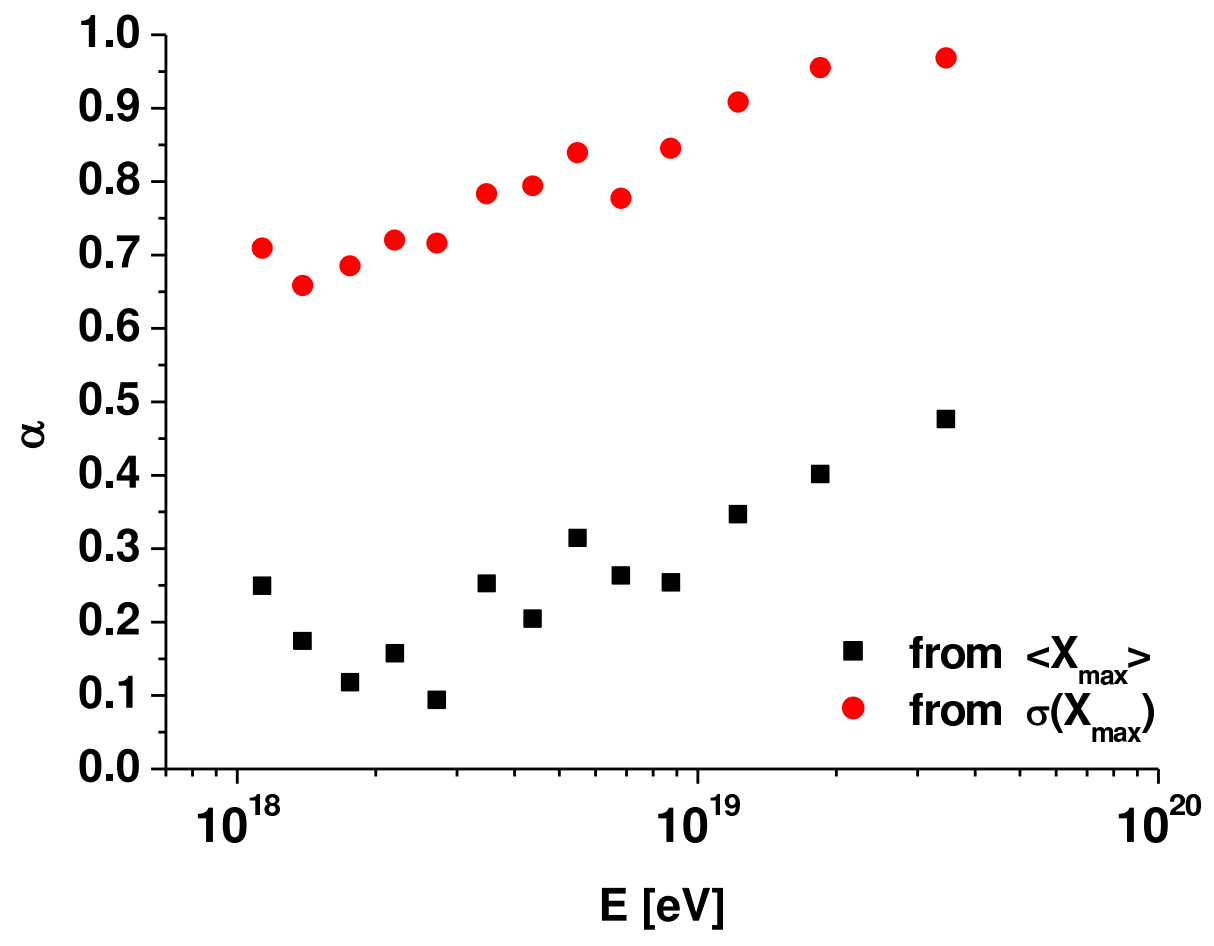

Figure 2. (Color online) The energy dependence of relative abundance of iron in $\mathrm{CR}$ as extracted from $\left\langle X_{\max }\right\rangle$ and $\sigma\left(X_{\max }\right)$ shown in Fig. 1 (in the frame of the QGSJETII model [4]).

and its fluctuations. The factor $k$ depends mainly on the way the energy dissipation takes place in the early stages of shower evolution and is particularly sensitive to the mean inelasticity and its fluctuations. Small fluctuations in multiplicity and $K$ result in smaller $k$ s .

In our case, in the absence of internal fluctuations, all showers would develop between the first interaction point and the maximum in the same amount of matter, $\Delta X=X_{\max }-X_{1}$, showing perfect correlation between the $X_{\max }$ and $X_{1}$. This means that their distributions should in this case have exactly the same shape, but shifted by $\Delta X$, i.e., the slope of the $X_{\max }$ distribution, $\Lambda$, should be equal to the mean interaction length $\lambda$. This relation will, however, be affected by some inevitable intrinsic fluctuations in shower development taking place after the first interaction $\|$. In this case, because of fluctuations in $\Delta X$, the correlation between $X_{\max }$ and $X_{1}$ will diminish. Roughly, one can write that

$$
\sigma\left(X_{\max }\right) \cong \sigma\left(X_{1}\right)+\xi[\sigma(\Delta X)]
$$

where $\sigma\left(X_{1}\right) \propto\left\langle X_{1}\right\rangle$ and the function $\xi(\sigma)$ describes the influence of shower fluctuations after the first (main) interaction point. Notice that for the probability distribution $\S$ Assuming similar fluctuations in multiplicity and inelasticity, a model predicting large $\langle n\rangle$ of secondary particles leads to a smaller overall fluctuations of the cumulative shower profile of secondaries, i.e., to smaller factor $k$ [5, 7].

II A comprehensive studies of the shower to shower fluctuations by means of Monte Carlo simulations can be found in recent works [9, 10]. 
given by Eq.(6) the fluctuation in $X_{1}$ is $\sigma\left(X_{1}\right)=\sqrt{\operatorname{Var}\left(X_{1}\right)}=\left\langle X_{1}\right\rangle=\lambda$. However, in the case when $X_{1}$ is interpreted as the main interaction point (in which the energy deposition to the shower is maximal) one would obtain a gamma distribution (instead of Eq. (6) ) for which $\sigma\left(X_{1}\right)=\sqrt{\operatorname{Var}\left(X_{1}\right)}=\left\langle X_{1}\right\rangle / \sqrt{\kappa}$, where $\kappa$ depends on the mean inelasticity, $\langle K\rangle$, and determines in which of the successive interactions of a projectile the energy deposition to the shower is maximalq. To summarize, because of Eq. (5), where $\left\langle X_{\max }\right\rangle=\left\langle X_{\max }^{e m}\right\rangle+\left\langle X_{1}\right\rangle$, we propose to use the following observable in which the influence of fluctuations of the first interaction is strongly suppressed,

$$
\left\langle X_{\max }\right\rangle-\sigma\left(X_{\max }\right) \cong\left\langle X_{\max }^{\max }[(E / A)(K /\langle n\rangle)]\right\rangle-\xi[\sigma(\Delta X)] .
$$

To test this observable, we first plot its energy behavior in Figs. 3 and 4 for the, respectively, Auger [1] and HiRes [2] data. Notice that now the HiRes data, where the distribution of $X_{\max }$ was truncated at $2 \sigma$ ( $\sigma_{T}$ denotes truncated fluctuations), show similar behavior as the Auger data. Notice also (cf. Fig. 3) that $\left\langle X_{\max }\right\rangle-\sigma\left(X_{\max }\right)$ given by Eq. (9) still depends on models of multiparticle production and is sensitive to the chemical composition of CR ( $p$ and Fe initiated showers are markedly different). Finally, Fig. 3 also tells us that the chemical composition cannot be the origin of the observation by Auger [1] that $\left\langle X_{\max }\right\rangle$ rises too slowly with energy and approximates the expectation for primary $F e$ nuclei. In fact, experimental data seem rather to be in fair agreement with the hypothesis of a proton dominant composition of the primary CR flux (assuming, of course, that the reference models used are roughly correct). Within the toy model of primary composition used before (with only two components: iron nuclei with relative abundance $\alpha$ and protons with abundance $1-\alpha$, cf. Eqs. (1) and (2)) we can again evaluate $\alpha$ as given by the Auger experiment but this time from $\left\langle X_{\max }\right\rangle-\sigma\left(X_{\max }\right)$. The result is shown in Fig. 5. For reference model QGSJETII, the abundance of iron is roughly independent of energy $(\alpha \approx 0.05 \div 0.1)$ and even for the model EPOS v.1.99 [1] leading to the maximal abundance of iron, $\alpha$ increases with energy rather slowly (remaining in the interval $\alpha \approx 0.15 \div 0.3$ ).

To summarize this part, we learn from Fig. 3 that the main contribution to the energy dependence of $\left\langle X_{\max }\right\rangle$ and $\sigma\left(X_{\max }\right)$ observed by Auger [1] comes from $\left\langle X_{1}\right\rangle$. This, however, can be affected by two factors: the cross section $\sigma_{\text {inel }}$ and inelasticity $K$ (in fact, not only by its mean value $\langle K\rangle$, but also by its distribution). Roughly, $\left\langle X_{1}\right\rangle=\lambda \cdot \kappa$, where $\kappa$ determines in which of the successive interactions of a projectile the energy deposition to the shower is maximal. For a uniform inelasticity distribution (in the maximal possible interval for a given $\langle K\rangle$ ), one has $\kappa \simeq 1+1.85(0.75-\langle K\rangle$ ). As shown recently in [12], if gluon saturation occurs in the nuclear surface region then $\sigma_{p-a i r}$ at $E>10^{18} \mathrm{eV}$ increases more rapidly with incident energy than is usually estimated. Although in [8, 13] we have argued for an overall decrease with energy of the inelasticity $K$, its increase at energies $E \approx 10^{18} \mathrm{eV}$ is by no means excluded + . Both possibilities

I Numerically for $\langle K\rangle \cong 0.7$ one has $\sigma\left(X_{1}\right) \cong 0.96\left\langle X_{1}\right\rangle$.

+ Recently the role of inelasticity in high energy CR was discussed in [14] using the percolation theory approach. 


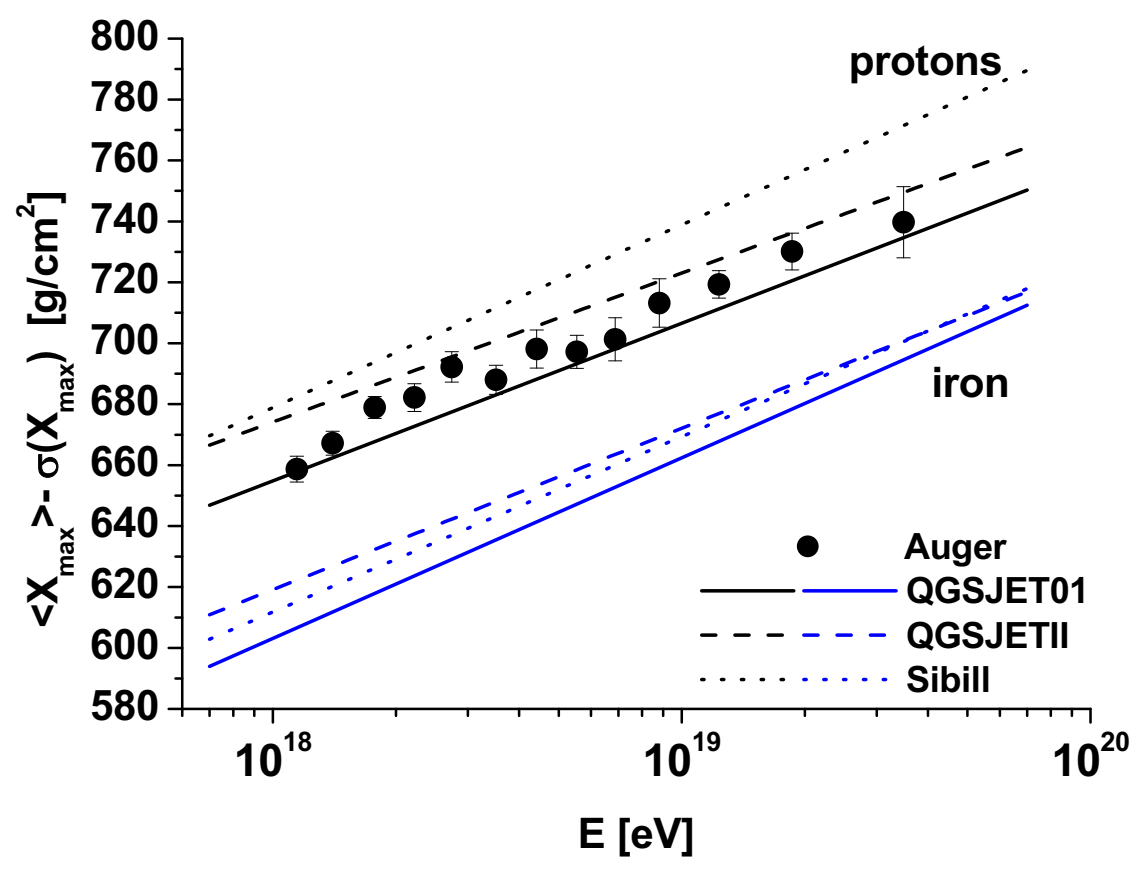

Figure 3. (Color online) $\left\langle X_{\max }\right\rangle-\sigma\left(X_{\max }\right)$ as deduced from the Auger data and compared to different models [1] for showers initiated by protons and iron. Note that experimental data prefer a proton composition.

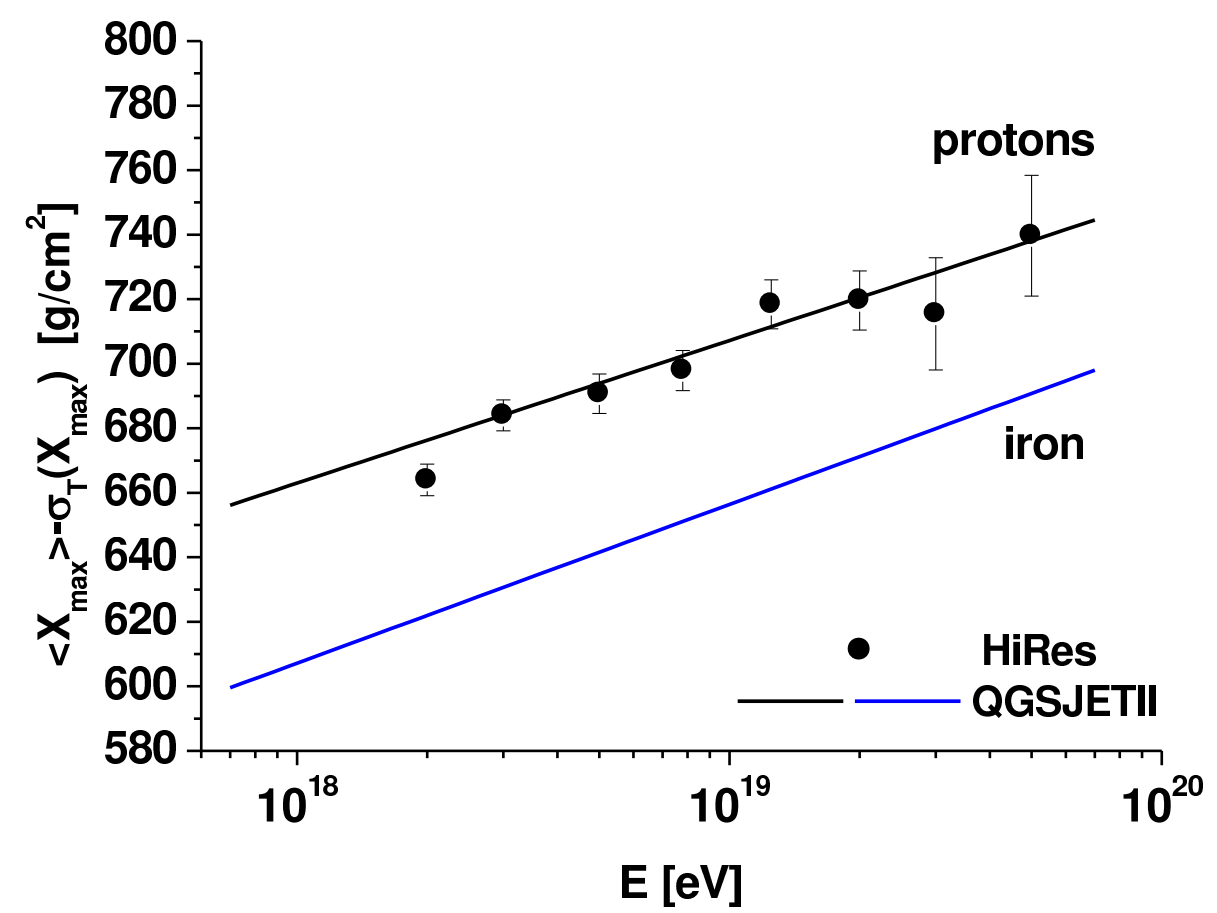

Figure 4. (Color online) The same as in Fig. 3, but for the HiRes experimental data (in this case data are truncated at $2 \sigma)[2]$.

require an abrupt onset of some "new physics" beyond the standard model, which would 


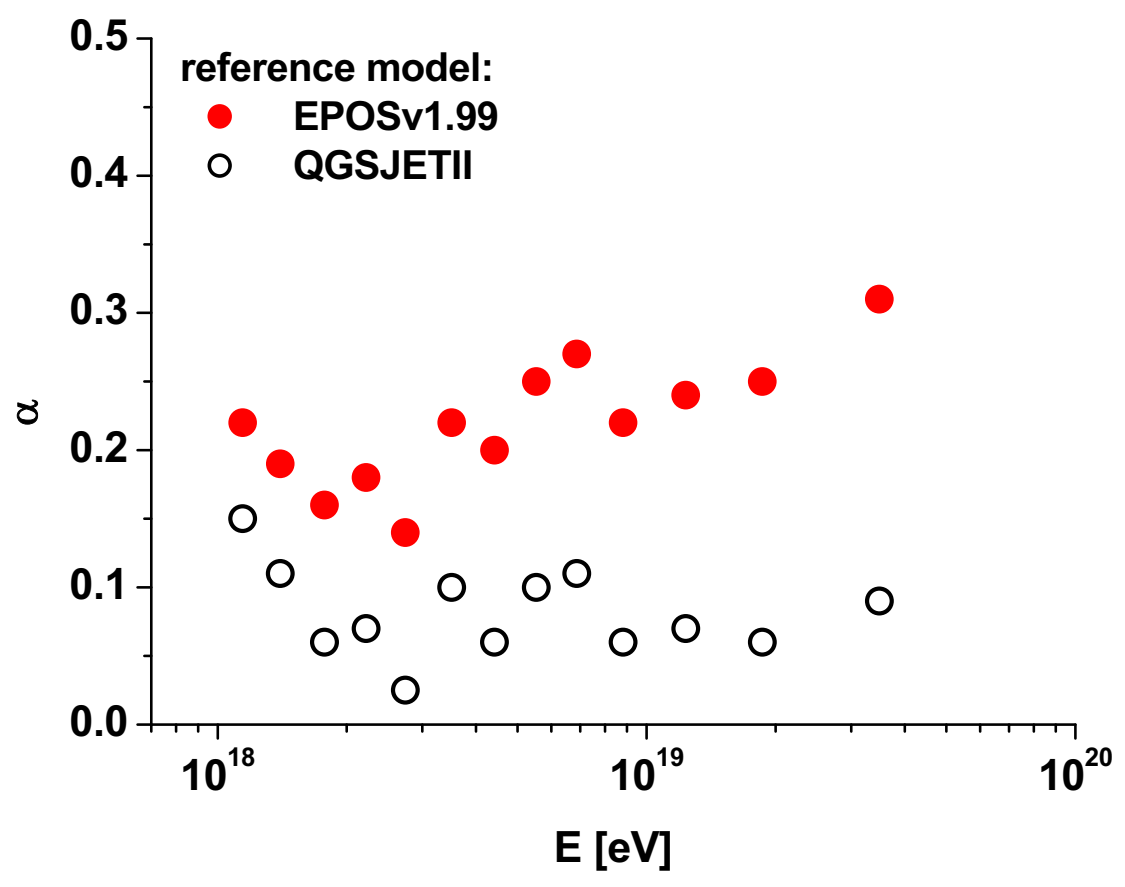

Figure 5. (Color online) The energy dependence of the relative abundance of iron in $\mathrm{CR}$ as extracted from $\left\langle X_{\max }\right\rangle-\sigma\left(X_{\max }\right)$ given by the Auger experiment and shown in Fig. 3 .

be difficult to accept. It is worth mentioning as an example the elongation rate, which in the case of Eq. (5) is given by [5, 15]

$$
D_{10}=\frac{d\left\langle X_{\max }\right\rangle}{d \log E}=\frac{X_{0}}{\log e}\left[1-\frac{d \log (A\langle n\rangle / K)}{d \log E}\right]+\frac{d\left\langle X_{1}\right\rangle}{d \log E} .
$$

As reported in [16], Auger observes the apparently abrupt change in $D_{10}$ at energy $\approx 2 \cdot 10^{18} \mathrm{eV}$, which could signal some new physics. If we denote $D_{10}^{\star}=$ $d\left[\left\langle X_{\max }\right\rangle-\sigma\left(X_{\max }\right)\right] / d \log E$, then one expects here something of the order of $D_{10}^{\star}-$ $D_{10}=-d \sigma\left(X_{\max }\right) / d \log E \cong 3.5 \mathrm{~g} \cdot \mathrm{cm}^{-2}$, depending on the increase in cross section adopted (chosen from existing models and predictions). However, the Auger data above $10^{18}$ provide the value $22 \mathrm{~g} \cdot \mathrm{cm}^{-2}$. It can be shown that such a large value leads to a very strong energy dependence of the cross section, $d \sigma_{p-a i r} / d \log E \cong 0.48 \sigma_{p-a i r}$, which seems at the moment to be very unrealistic and contradicts even the scenario of gluon saturation on the nuclear surface recently proposed in [12. A more detailed discussion of this problem is outside the scope of this note. On the other hand, the center of mass collision energies of the order of few hundred $\mathrm{TeV}$ observed here are well beyond those to be studied in the foreseeable future at LHC. This means that CR are, most probably, the only future source of information on the properties of interactions at these energies and surprises should not be ruled out a priori.

As mentioned above the Hires data (which are truncated at 2 $\sigma$ ) [2] show similar 


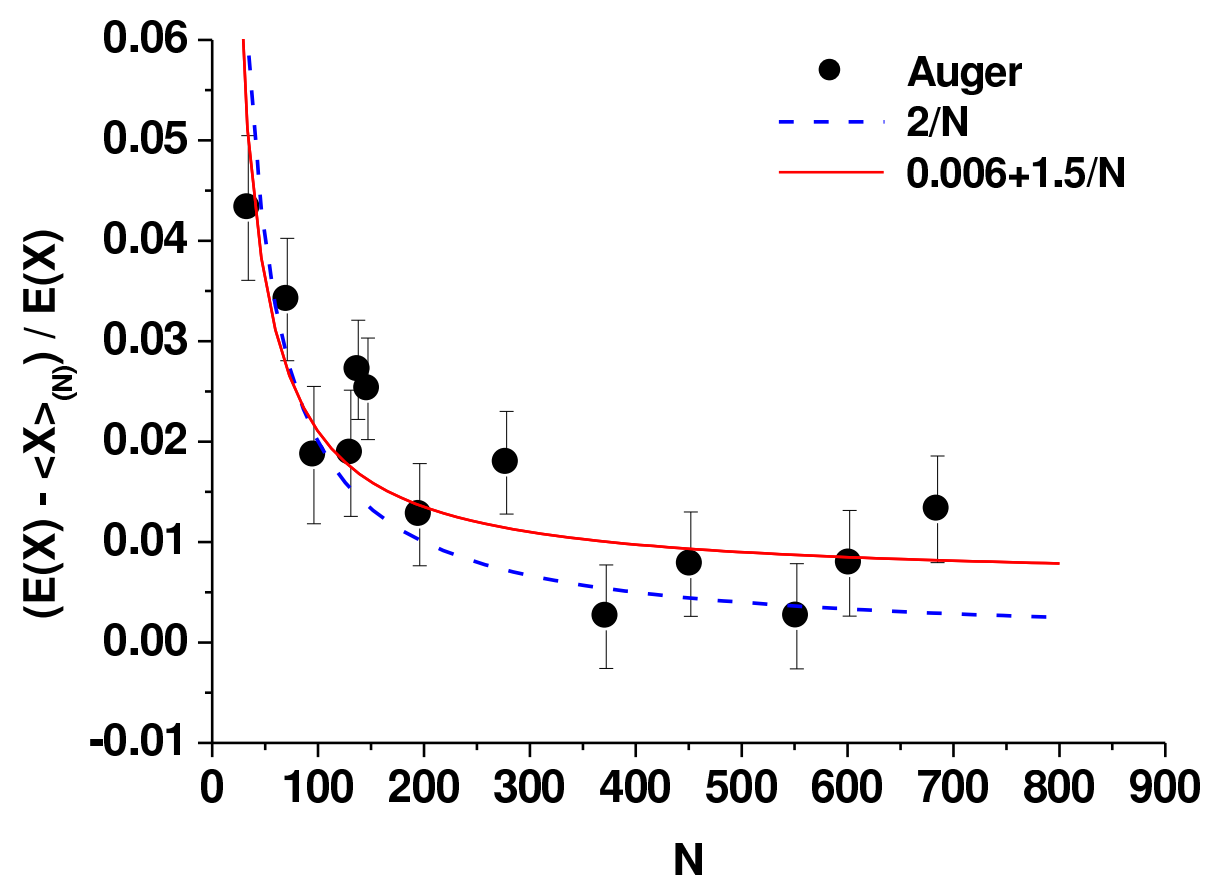

Figure 6. (Color online) Illustration of the effect of statistics as given by Eq. (14) with $\langle X\rangle_{(\infty)}=E(X)$ assumed to be the same as the mean value given by the QGSJET01 model [17, see text for details.

energy dependence for $\left\langle X_{\max }\right\rangle-\sigma\left(X_{\max }\right)$ as the Auger data, c.f. Fig. 4, This indicates that, in both cases, the crucial factors are the tails of the $X_{\max }$ distributions. For a small sample, the values of $X_{\max }$ near the maximum of the distribution are preferred, and the estimated mean value $\left\langle X_{\max }\right\rangle$ differs from the expected value, $E\left(X_{\max }\right)$. To investigate whether the effect observed by Auger could be connected to small statistics, notice that, because the distribution of distances of the first interaction is $\exp (-X / \lambda)$, when calculating $\langle X\rangle=\frac{1}{N} \sum_{i}^{N} X_{i}$ one encounters $S=\sum_{i}^{N} X_{i}$ which has a gamma distribution,

$$
p(S)=\frac{S^{N-1}}{\lambda^{N} \Gamma(N)} \exp \left(-\frac{S}{\lambda}\right)
$$

For small samples one in fact observes the most probable values, which for a gamma distribution is equal to $S^{(\max )}=\lambda(N-1)$. This means that we can expect that, for a sample consisting with $N$ elements one has

$$
\langle X\rangle_{(N)}=\frac{1}{N} \sum_{i}^{N} X_{i} \approx \frac{1}{N} S^{(\max )}=\lambda\left(1-\frac{1}{N}\right),
$$

whereas the true expectation values for the exponential distribution (obtained for $N \rightarrow \infty)$ is $E(X)=\lambda$. Therefore, for a sample of $N$ elements the estimator $\langle X\rangle_{N}$ is biased by a value of the order of

$$
E(X)-\langle X\rangle_{(N)} \approx \frac{\lambda}{N}
$$


In Fig. 6] we compare

$$
\frac{\langle X\rangle_{(\infty)}-\langle X\rangle_{(N)}}{\langle X\rangle_{(\infty)}}=1-\frac{\langle X\rangle_{(N)}}{\langle X\rangle_{(\infty)}} \propto \frac{1}{N}
$$

with data of Auger, here $\langle X\rangle_{(\infty)}=E(X)$ denotes the value given by the model. Because the reference model (here QGSJET01) does not exactly describe the experimental data (even in the lower energy region, i.e. for large values of $N$ ) we use here the simple formula $a+b / N$ to describe the dependence on $N$. To further illustrate the significance of tails of $X_{\max }$ distributions, we examine the truncated $X_{\max }$ distribution in the interval $\left(0, X_{c u t}\right)$, cf., Fig. 7 where $\left\langle X_{\max }\right\rangle, \sigma\left(X_{\max }\right)$ and $\left\langle X_{\max }\right\rangle-\sigma\left(X_{\max }\right)$, evaluated for different $X_{c u t}$, are presented. Notice that $\left\langle X_{\max }\right\rangle$ and $\sigma\left(X_{\max }\right)$ are strongly dependent on the value of $X_{\text {cut }}$. On the other hand, their difference introduced in Eq. (9) above, $\left\langle X_{\max }\right\rangle-\sigma\left(X_{\max }\right)$, is rather insensitive to the cutting procedure used. Therefore, the possible biasses of the tail of the $X_{\max }$ distribution do not influence this observable.

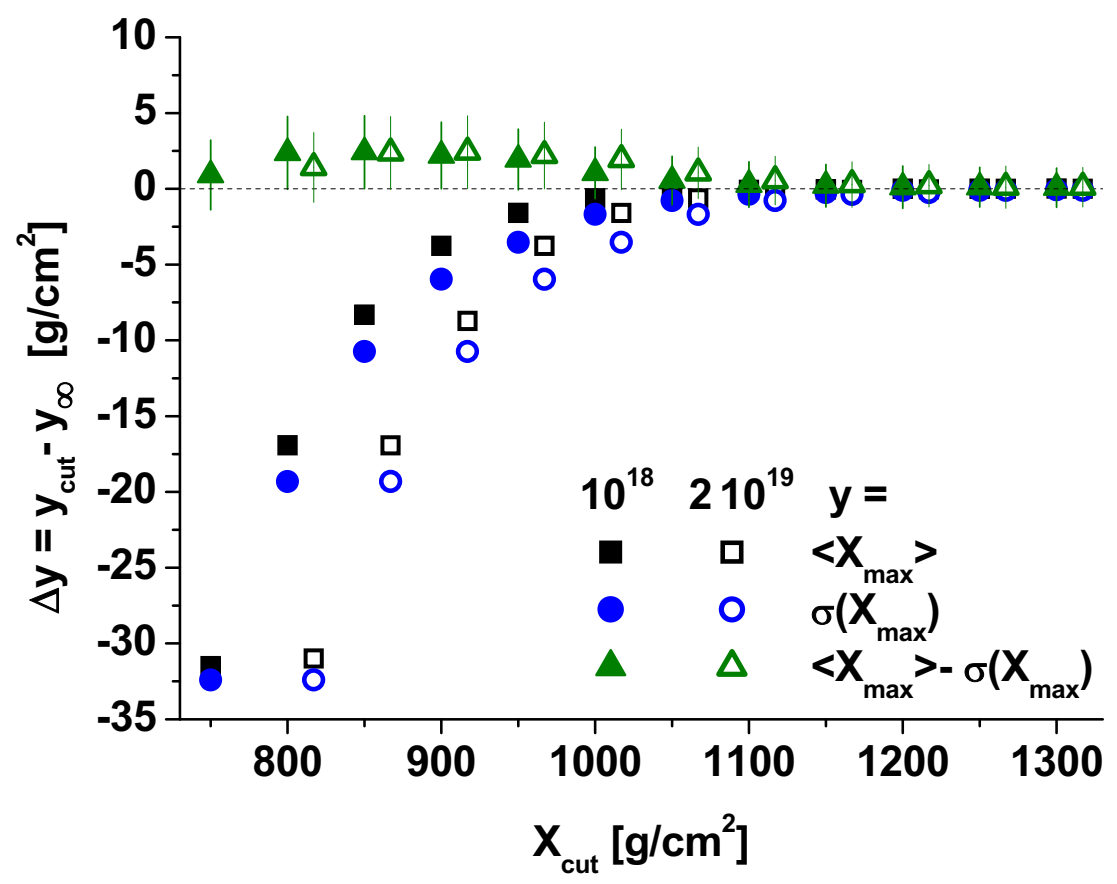

Figure 7. (Color online) Difference of the observable $y_{\text {cut }}$, evaluated from the $X_{\max }$ distribution truncated at $X_{c u t}$, and $y_{\infty}$, evaluated from the unbiased distribution (given by QGSJET01 model for primary protons with energy $10^{18} \mathrm{eV}$ and $2 \cdot 10^{19}$ $\mathrm{eV}$. When energy increases, the observed dependence shifts towards the higher $X_{c u t}$, proportionally to the increase of $\left\langle X_{\max }\right\rangle$ with energy, i.e., the dependence of $\Delta y$ on $X_{c u t}-\left\langle X_{\max }\right\rangle_{\infty}$ remains roughly the same for all primary energies.

In conclusion, we argue that the spectacular energy dependence of the shower maxima distribution reported by the Auger collaboration [1] is not necessarily (or not only) due to the changes of chemical composition of primary cosmic rays. The observed effect (or, at least, a substantial part of it) seems rather to be caused by the unexpected 
changes of the depth of first interaction, $X_{1}$. However, the energy dependence of $\left\langle X_{1}\right\rangle$ can be affected by a rapid increase of cross section and/or increase of inelasticity in energies above $2 \cdot 10^{18} \mathrm{eV}$. Both possibilities require an abrupt onset of some "new physics" in this energy region and are therefore questionable. The HiRes data [2], where

the $X_{\max }$ distribution was truncated and, after that operation, is consistent with the proton spectrum, brings in the possible role of biases of the $X_{\max }$ distribution indicating that the ways of analyzing CR data of highest energy still remains an open problem. We argue that it would be highly desirable to analyze the observable $\left\langle X_{\max }\right\rangle-\sigma\left(X_{\max }\right)$ (cf. Eq. (9) ) in which fluctuations of the depth of the first interaction, as well as the possible biases of the tail of $X_{\max }$ distribution, are strongly suppressed. This observable still depends on the model of multiparticle production and is sensitive to the chemical composition of the primary CR.

Summarizing, though the problem of the chemical composition of CR seem still unresolved, we expect that the large spread observed in the results can find an explanation by comparing the different sensitivities to the composition of $\mathrm{CR}$ in various observables. A deeper understanding, both of the hadronic interaction models and of the systematics of data analysis, which could bias the results, is also needed. Such a detailed analysis, with detailed simulation studies, is, however, outside the scope of this paper.

\section{Acknowledgment}

Partial support (GW) of the Ministry of Science and Higher Education under contract DPN/N97/CERN/2009 is gratefully acknowledged. We would like to thank warmly dr Eryk Infeld for reading this manuscript.

\section{References}

[1] Abraham J et al. (Pierre Auger Collaboration) 2010 Phys. Rev. Lett. 104091101

[2] Abbasi R U et al., (The High Resolution Fly's Eye Collaboration) 2010 Phys. Rev. Lett. 104 161101

[3] Schwarzschild B 2010 Phys. Today 6315

[4] Ostapchenko S S 2006 Nucl. Phys. B Proc. Suppl. 151143

[5] Alvarez-Muniz J, Engel R, Gaisser T K, Ortiz J A, and Stanev T 2002 Phys. Rev. D 66033011 and 2004 Phys. Rev. D 69103003

[6] Risse M 2004 Acta Phys. Polon. B 351787

[7] Ulrich R, Blümer J, Engel R, Schüssler F, and Unger M 2009 New J. Phys. 11065018

[8] Włodarczyk Z 1993 J. Phys. G 19 L133

[9] Hansen P M, Alvarez-Muniz J, and Vazquez R A, 2011 Astrop. Phys. 34503

[10] Ulrich R, Engel R, and Unger M, 2011 Phys. Rev. D 83054026

[11] Pirog T, and Werner K 2008 Phys. Rev. Lett. 171101

[12] Portugal L, and Kodama T 2010 Nucl. Phys. A 8371

[13] Shabelski Yu M, Weiner R M, Wilk G, and Włodarczyk Z 1992 J. Phys. G 181281

[14] Dias de Deus J, Esprito Santo M C, Pimenta M, and Pajares C 2006 Phys. Rev. Lett. 96162001

[15] Lindsley J, and Watson A A 1981 Phys. Rev. Lett. 46459 
[16] Unger M (for the Pierre Auger Coll.) 2009 Nucl. Phys. B (Proc. Suppl.) 190, 240

[17] Kalmykov N N, and Ostapchenko S S 1993 Phys. At. Nucl. 56346 